\title{
Spline-based image-to-volume registration for three-dimensional electron microscopy
}

\author{
S. Jonici ${ }^{\mathrm{a}, \mathrm{e}, *}$, C.O.S. Sorzano ${ }^{\mathrm{a}, \mathrm{b}}$, P. Thévenaz ${ }^{\mathrm{a}}$, C. El-Bez ${ }^{\mathrm{c}}$, \\ S. De Carlo ${ }^{\mathrm{d}}$, M. Unser ${ }^{\mathrm{a}, 1}$ \\ ${ }^{a}$ Biomedical Imaging Group, École polytechnique fédérale de Lausanne (EPFL), CH-1015 Lausanne VD, Switzerland \\ ${ }^{\mathrm{b}}$ Escuela Politécnica Superior, Universidad San Pablo-CEU, Campus Urb. Montepríncipe s/n, 28668 Boadilla del Monte, Madrid, Spain \\ ${ }^{\mathrm{c}}$ Laboratoire d'Analyse Ultrastructurale, Université de Lausanne, CH-1015 Lausanne VD, Switzerland \\ ${ }^{\mathrm{d}}$ Howard Hughes Medical Institute, Molecular and Cell Biology Department, University of California, Berkeley, CA 94720-3200, USA \\ ${ }^{\mathrm{e}}$ Laboratoire de Minéralogie-Cristallographie Paris de Paris 6, Université Pierre et Marie Curie, Campus BOUCICAUT, Case 115, \\ 140, rue de Lourmel, F-75005 Paris, France
}

Received 3 December 2003; received in revised form 8 February 2005; accepted 16 February 2005

\begin{abstract}
This paper presents an algorithm based on a continuous framework for a posteriori angular and translational assignment in three-dimensional electron microscopy (3DEM) of single particles. Our algorithm can be used advantageously to refine the assignment of standard quantized-parameter methods by registering the images to a reference 3D particle model. We achieve the registration by employing a gradient-based iterative minimization of a least-squares measure of dissimilarity between an image and a projection of the volume in the Fourier transform (FT) domain. We compute the FT of the projection using the central-slice theorem (CST). To compute the gradient accurately, we take advantage of a cubic B-spline model of the data in the frequency domain. To improve the robustness of the algorithm, we weight the cost function in the FT domain and apply a "mixed" strategy for the assignment based on the minimum value of the cost function at registration for several different initializations. We validate our algorithm in a fully controlled simulation environment. We show that the mixed strategy improves the assignment accuracy; on our data, the quality of the angular and translational assignment was better than 2 voxel (i.e., $6.54 \AA$ ). We also test the performance of our algorithm on real EM data. We conclude that our
\end{abstract}

\footnotetext{
*Corresponding author. Laboratoire de Minéralogie-Cristallographie Paris de Paris 6, Université Pierre et Marie Curie, Campus BOUCICAUT, Case 115, 140, rue de Lourmel, F-75005 Paris, France. Tel.: + 33144275241 ; fax: + 33144273785 .

E-mail addresses: Slavica.Jonic@1mcp.jussieu.fr (S. Jonić), michael.unser@epfl.ch (M. Unser).

URL: http://bigwww.epfl.ch/.

${ }^{1}$ Also for correspondence.
} 
algorithm outperforms a standard projection-matching refinement in terms of both consistency of 3D reconstructions and speed.

(C) 2005 Elsevier B.V. All rights reserved.

Keywords: 2D/3D registration; CST; Splines; 3DEM; Angular assignment

\section{Introduction}

Three-dimensional (3D) electron microscopy (EM) comprises methods that facilitate the visualization of biological structures with sizes ranging from $100 \AA$ to $1 \mu[1-4]$. A 3D model of a particle is usually computed from its EM images in a twostep procedure. First, the in-plane coordinates of the center of the particle as well as its in-plane and out-of-plane rotations are determined and assigned to each particle image [5-18]. Second, a reconstruction algorithm is applied to compute the 3D model using the particle images and the previous angular and translational assignment [19-21]. Sometimes, the two steps are performed simultaneously $[22,23]$.

We can classify all the techniques for the angular assignment into two groups: referencefree $[5,6,8,9,12,14,16,18]$ and reference-based $[7,10,11,13,15,17]$. The goal of the reference-based methods is to refine a particle assignment with respect to a reference volume. The operation of positioning an image plane with respect to a volume is known as image-to-volume registration. The goal of the new assignment is to improve the previous 3D reconstruction. As the goal of reference-free methods is to build a volume from scratch, we do not discuss them any further, but we take for granted the existence of an imperfect reference that we want to refine.

The standard reference-based techniques $[13,15,17]$ determine the center and the orientation of the particles by a set of quantized parameters. Penczek et al. [13] and Sorzano et al. [17] assign these parameters based on the cross-correlation between the EM images and a library of projections of the reference volume. The cross-correlation is computed using the two-dimensional (2D) Fourier transforms (FTs) of the EM images and of the library images [13] or using their 2D wavelet transforms [17]. We refer to the former method as projection matching (PM), and to the latter as multiresolution projection matching (MPM). By contrast, Radermacher [15] performs the assignment relying on the cross-correlation between the $2 \mathrm{D}$ radon transform (RT) of each EM image and the $3 \mathrm{D}$ RT of the volume. We refer to this method as radon assignment (RA). The advantage of the latter method is that the 3D RT contains the same information as a discrete set of all possible volume projections but it is represented using a smaller data set. These quantized-parameter methods apply exhaustive-search techniques to determine the optimal parameters. Their main drawback is that the quality of the registration depends on the quantization step. The smaller is the step, the more accurate, but slower is the parameter assignment.

In our previous work, we have developed an algorithm that registers a computed-tomography volume to a set of fluoroscopy images by assigning a set of continuous orientational and translational parameters to the images [24,25]. This algorithm performs the registration in the space domain. In this paper, we present its FT equivalent applied to a posteriori angular and translational assignment in 3DEM. To determine the center and the orientation of the particles by a set of continuous parameters, it employs a Levenberg-Marquardt (LM) minimization of a least-squares measure of dissimilarity between the 2D FT of the EM image and the extracted corresponding central slice of the 3D FT of the volume based on the central-slice theorem (CST).

The two algorithms that are the most similar to ours are that of Provencher and Vogel [22,23] and FREALIGN [10] which also compute continuous parameters of the particle orientation and position. The method of Provencher and Vogel $[22,23]$ performs the parameter refinement and the 3D reconstruction simultaneously. It models the 3D FT of the electron density of the particle by a truncated expansion in a complete orthonormal 
set of basis functions in spherical polar coordinates. The data are compressed, and the expansion coefficients together with the parameters of the particle orientations are estimated for all particles simultaneously. The estimation is accomplished using a modified Gauss-Newton minimization of a weighted variance of the CST-based fit of the 2D FT of each particle image into the 3D model. Their method optimizes a large number of parameters, which means a higher risk for the algorithm to get trapped into local optima of the cost function, and an augmented dependence on the quality of the starting estimates for the parameters. In contrast, FREALIGN [10] and our algorithm have an easier parameter control since they estimate 5 parameters per particle only, for each particle independently. Another advantage of these algorithms is their versatility since they can be applied in combination with any available reconstruction algorithms within a procedure for iterative refinement of the estimated parameters (Fig. 1). However, while FREALIGN minimizes the phase dissimilarity between the FT of the experimental image and its model weighted by the amplitude of the FT of the experimental image, our algorithm minimizes both the amplitude dissimilarity between the FT of the experimental image and its model, and the phase one. Both algorithms use a 3D reference

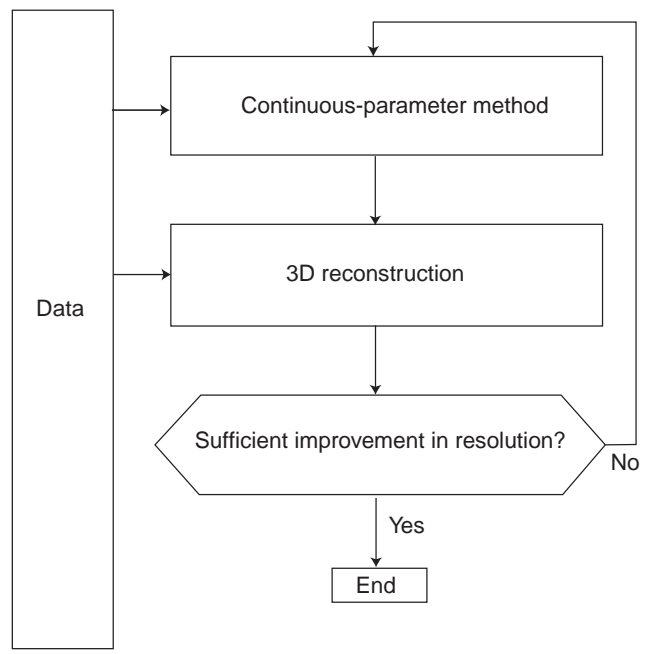

Fig. 1. Refinement of the 3D model of a particle using continuous-parameter image-to-volume registration. model that is created from electron micrographs of the particles. In return, they require interpolation of the 3D FT of the volume.

Contrary to FREALIGN which uses the FT of a box for the interpolation process, our algorithm uses a cubic B-spline model [26-29] of the volume in the FT domain for an accurate extraction of the slices of the 3D FT as well as for having the gradient of the dissimilarity measure well-defined everywhere. This is a necessary condition for an accurate assignment of the particle orientations and positions in the space of continuous parameters. This interpolation scheme allows us to take advantage of the powerful LM optimization method that is faster than Powell's method of FREALIGN since we have access to the gradient of the cost function. We contribute also to the improvement of the algorithm robustness.

Like all iterative algorithms, our method requires an initial guess for the unknown parameters. To reduce the sensitivity to the choice of the initial parameters, we have developed a novel strategy for the assignment that launches the algorithm for a number of initial guesses and, then, selects the best starting point for each experimental image. As criterion for the selection of the best initialization, we use the minimum value of the dissimilarity measure at registration. The advantage of this strategy is in avoiding "bad" starting points, which improves the registration robustness and accuracy. We also weight the dissimilarity measure such that all frequencies are attenuated, particularly the high ones while the DC component is discarded. In this paper, we show the performance of our algorithm when using this strategy based on three starting points. More precisely, we initialize the algorithm using the assignments by MPM, PM, and RA (Fig. 2). A generalization to any number of points is straightforward.

The paper is organized as follows: we describe the geometry of the problem in Section 2. The basic elements of our frequency-based algorithm are given in Section 3. In Section 4, we validate the algorithm using 3D models of proteins from the Protein Data Bank [30] (PDB) (http://www. rcsb.org/pdb/) and synthesized EM images of these proteins. These images have a known 


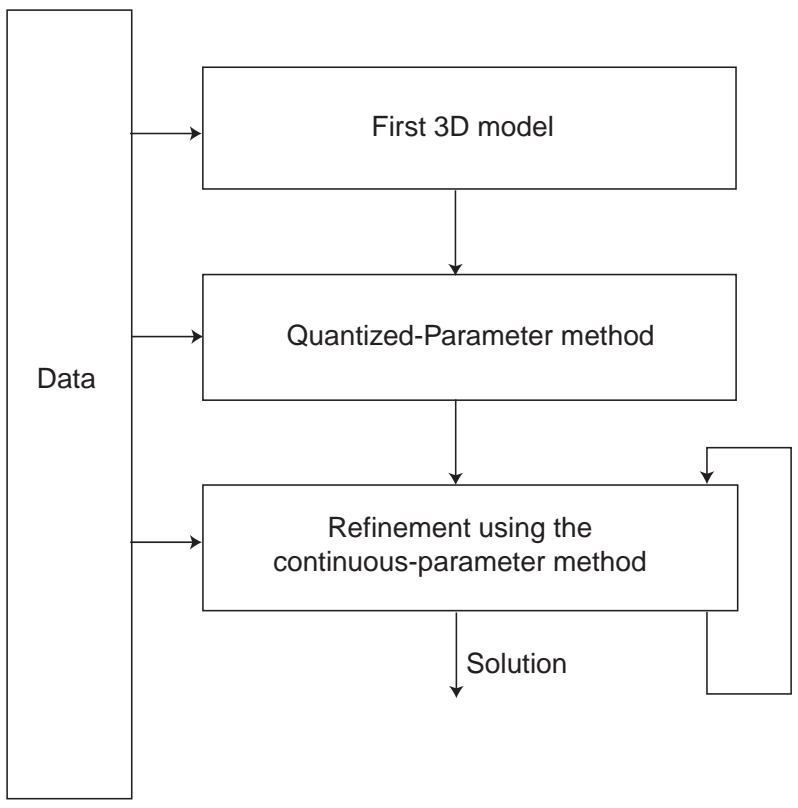

Fig. 2. Initialization by a standard quantized-parameter method.

ground-truth alignment with respect to the 3D protein model. This allows an objective measure of the assignment accuracy. We also have at our disposal a set of experimental EM images and a 3D model of a protein for which we do not know the ground-truth alignment. The performance of the algorithm using such data is investigated in Section 5. The conclusions are given in Section 6.

\section{Geometrical setup}

We define the volume orientational and translational parameter $\mathbf{a}=\left(a_{1}, a_{2}, \ldots, a_{6}\right)$ in the reference coordinate system (R-COS) by three rotations and three translations of the volume. Let us denote the system of 3D voxel indexes $\mathbf{m}=$ $\left(m_{1}, m_{2}, m_{3}\right) \in \mathbb{R}^{3}$ by the voxel coordinate system $(\mathrm{V}-\mathrm{COS})$ and the system of $2 \mathrm{D}$ pixel indexes $\mathbf{k}=$ $\left(k_{1}, k_{2}, 0\right) \in \mathbb{R}^{2} \times\{0\}$ by the pixel coordinate system (P-COS). We rotate the volume by applying a $3 \times 3$ rotation matrix $R$ to the $\mathrm{V}-\mathrm{COS}$ coordinate of each voxel of the volume. This matrix is determined by three Euler angles: $\varphi, \theta$, and $\psi$, that is, $R=R_{z}(\psi) R_{y}(\theta) R_{z}(\varphi)$, where $R_{x}(\alpha)$ indi- cates the matrix of rotation around the $x$-axis by the angle $\alpha$. The volume translation is described by a vector $\mathbf{t}=(\Delta x, \Delta y, \Delta z)$, where $\Delta x, \Delta y$, and $\Delta z$ are translations along the $x$ -,$y$-, and $z$-axes. We transform the V-COS coordinate $\mathbf{m}$ of a point of the volume into its corresponding P-COS coordinate $\mathbf{k}=\Lambda_{1} H\left(R \Lambda_{2} \mathbf{m}+\mathbf{t}\right)$, where $\Lambda_{1}$ and $\Lambda_{2}$ are diagonal scaling matrices, and where $H$ projects a $3 \mathrm{D}$ vector onto the $x y$-plane, with

$$
H=\left(\begin{array}{lll}
1 & 0 & 0 \\
0 & 1 & 0 \\
0 & 0 & 0
\end{array}\right)
$$

Similarly, we transform the P-COS coordinate of a point $\mathbf{k}$ inside the image into a line of corresponding V-COS coordinates $\mathbf{n}_{0}+\xi \mathbf{n}$, where $\xi \in \mathbb{R}$ is some free scalar parameter, with $\mathbf{n}_{0}=\Lambda_{2}^{-1} R^{-1}\left(\Lambda_{1}^{-1} \mathbf{k}-\mathbf{t}\right)$, $\mathbf{n}=\Lambda_{2}^{-1} R^{-1} \Lambda_{1}^{-1} \mathbf{e}_{3}$, and $\mathbf{e}_{3}=(0,0,1)$.

Given an EM image and a 3D model of a particle, our algorithm assigns a set of orientational and translational parameters a to the corresponding image plane in the R-COS such that the volume projection onto the plane is the 
most similar to the given image. This is equivalent to positioning the volume in the R-COS with respect to a fixed image plane. We repeat the same assignment procedure for each image from a set of given EM images. We compute a by minimizing the cost function which is a real-valued function of five variables $a_{i}, i=1,2, \ldots, 5$ (the out-of-plane and in-plane rotation angles, and the in-plane translations).

Note that, in this application, we do not optimize the cost function in all six unknown parameters but in only five of them. These parameters are $\mathbf{a}=(\varphi, \theta, \psi, \Delta x, \Delta y)$. We cannot recover $\Delta z$ because the parallel-beam projection geometry is invariant with respect to the shift along the projection direction (here, the $z$-axis).

\section{Algorithm}

Our frequency-based algorithm is summarized in Fig. 3. The input data for the algorithm are: (1) the 3D model of the particle, (2) the EM images of the particle, (3) the pixel and voxel sizes, and (4) the initial angular and translational assignment for the particle images.

This method minimizes the dissimilarity between the FTs of the EM images and the extracted corresponding central slices of the 3D discrete FT (DFT) of the volume; the extraction is performed according to the CST approach given in Section 3.1. The required interpolation in the frequency domain is described in Section 3.2. The weighting of the dissimilarity measure for the robustness improvement is shown in Section 3.3. The optimization algorithm is presented in Section 3.4.

Error-free (sinc-based) interpolation in the FTdomain can be achieved if the data are spacelimited, which is not always the case. To interpolate the data in the FT-domain accurately enough, we assume that they are nearly spacelimited. To enforce this assumption, we perform a spatial apodization of the input volume and images by Gaussian windows with the appropriate standard deviations. The apodization of the input volume and images by Gaussian windows is represented by the two "Windowing" blocks in Fig. 3.

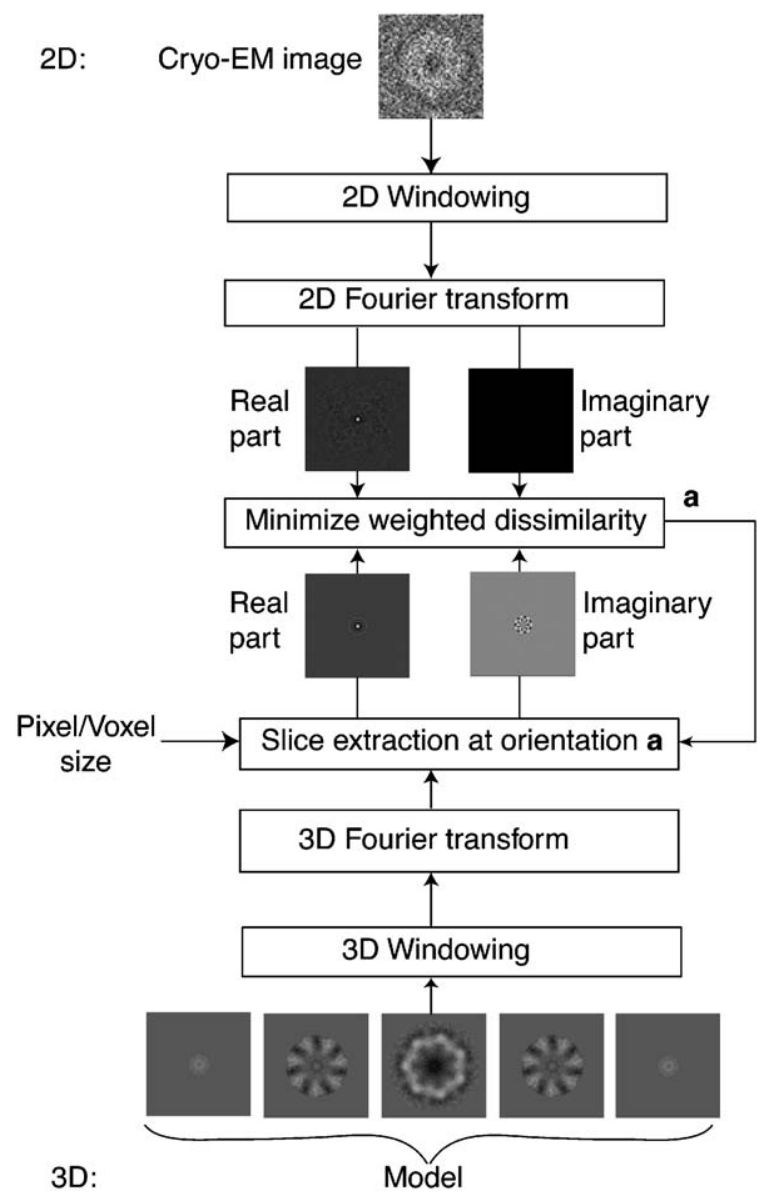

Fig. 3. Continuous angular and translational assignment in the frequency domain.

\subsection{CST approach}

Given a volume $f$ whose orientation and translation in the R-COS is described by a (Fig. 4), a projection of the volume along the ray determined by the unit vector $\mathbf{e}_{3}$ through the image-plane point with the P-COS coordinate $\mathbf{k}=$ $\left(k_{1}, k_{2}, 0\right)$ is as follows:

$p_{\mathbf{a}}(\mathbf{k})=\int_{\mathbb{R}} f\left(\mathbf{n}_{0}+\xi \mathbf{n}\right) \mathrm{d} \xi$.

Let us write the 2D FT of the projection $p_{\mathbf{a}}$ as

$\hat{p}_{\mathbf{a}}(\boldsymbol{\omega})=\int_{\mathbb{R}^{2}}\left(\int_{\mathbb{R}} f\left(\mathbf{n}_{0}+\xi \mathbf{n}\right) \mathrm{d} \xi\right) \mathrm{e}^{-\mathrm{j}\langle\boldsymbol{\omega}, \mathbf{k}\rangle} \mathrm{d}^{2} k$, 


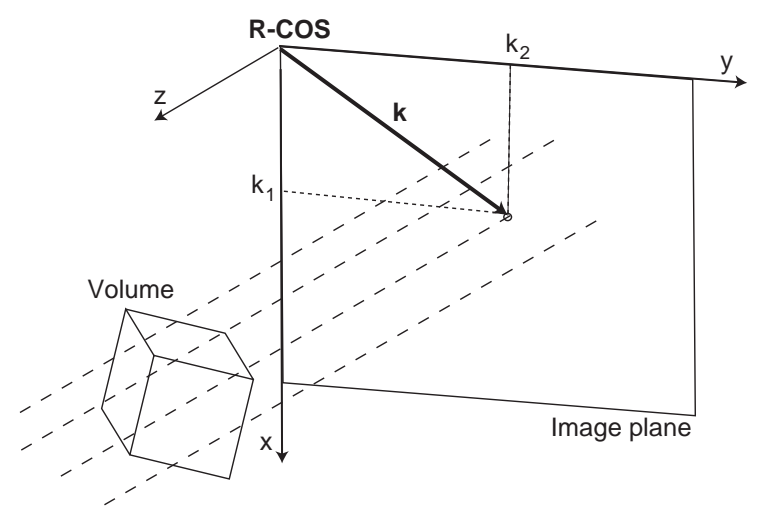

Fig. 4. Parallel-beam X-ray geometry.

where $\boldsymbol{\omega}=\left(\omega_{1}, \omega_{2}, 0\right) \in \mathbb{R}^{2} \times\{0\}$ is a spatial-frequency vector and where $\langle\mathbf{x}, \mathbf{y}\rangle=\mathbf{x}^{\top} \mathbf{y}$ stands for the inner product between the vectors $\mathbf{x}$ and $\mathbf{y}$. Recalling the definitions of $\mathbf{n}_{0}$ and $\mathbf{n}$, we can rewrite (3) as

$\hat{p}_{\mathbf{a}}(\boldsymbol{\omega})=\int_{\mathbb{R}^{3}} f\left(\Lambda_{2}^{-1} R^{-1}\left(\Lambda_{1}^{-1} \mathbf{r}-\mathbf{t}\right)\right) \mathrm{e}^{-\mathrm{j}\langle\boldsymbol{\omega}, \mathbf{r}\rangle} \mathrm{d}^{3} r$,

where $\mathbf{r}=\mathbf{k}+\xi_{\mathbf{e}_{3}}$. Let us perform the change of variable $\mathbf{s}=\Lambda_{2}^{-1} R^{-1}\left(\Lambda_{1}^{-1} \mathbf{r}-\mathbf{t}\right)$, which leads to

$$
\begin{aligned}
\hat{p}_{\mathbf{a}}(\boldsymbol{\omega})= & \left|\operatorname{det} \Lambda_{1} \Lambda_{2}\right| \int_{\mathbb{R}^{3}} f(\mathbf{s}) \mathrm{e}^{-\mathrm{j}\left\langle\boldsymbol{\omega}, \Lambda_{1}\left(R \Lambda_{2} \mathbf{s}+\mathbf{t}\right)\right\rangle} \mathrm{d}^{3} s \\
= & \left|\operatorname{det} \Lambda_{1} \Lambda_{2}\right|\left(\int_{\mathbb{R}^{3}} f(\mathbf{s}) \mathrm{e}^{-\mathrm{j}\left\langle\left(\Lambda_{1} R \Lambda_{2}\right)^{\top} \boldsymbol{\omega}, \mathbf{s}\right\rangle} \mathrm{d}^{3} s\right) \\
& \times \mathrm{e}^{-\mathrm{j}\left\langle\boldsymbol{\omega}, \Lambda_{1} \mathbf{t}\right\rangle},
\end{aligned}
$$

where the term in parentheses represents the 3D FT $\hat{f}$ of the volume $f$ evaluated at the 3D frequency $\left(\Lambda_{1} R \Lambda_{2}\right)^{\top} \boldsymbol{\omega}$. The $2 \mathrm{D}$ FT of the projection $p_{\mathrm{a}}$ is therefore given by

$\hat{p}_{\mathbf{a}}(\boldsymbol{\omega})=|\operatorname{det} \Lambda| \hat{f}\left(\left(\Lambda_{1} R \Lambda_{2}\right)^{\top} \boldsymbol{\omega}\right) \mathrm{e}^{-\mathrm{j}\left\langle\boldsymbol{\omega}, \Lambda_{1} \mathbf{t}\right\rangle}$,

where $\Lambda=\Lambda_{1} \Lambda_{2}$.

Eq. (6) is a matrix form of the CST suited to our parameterization. It tells us how we can obtain the 2D FT of a projection with the orientational and translational parameters a using the 3D FT of the volume. It comprises the extraction of the central slice at orientation determined by $\Lambda_{1} R \Lambda_{2}$ from the 3D FT, the slice phase shift determined by $\Lambda_{1}$ and t, and the slice scaling given by $|\operatorname{det} \Lambda|$. Since we have discrete data at our disposal, we replace the
FT by the DFT. The slice extraction involves the interpolation of the real and imaginary parts of the 3D DFT of the volume. We model each part of the complex 3D DFT by a continuous B-spline 3D data model presented below.

\subsection{Data interpolation}

Given the discrete samples $\hat{x}_{\mathbf{n}}=\hat{x}(\mathbf{n}), \mathbf{n}=$ $\left(n_{1}, \ldots, n_{N}\right) \in \mathbb{Z}^{N}$, of the FT of a function $x$, we can reconstruct some continuously defined FT of a function $y$ using the following B-spline model:

$$
\begin{aligned}
\hat{y}(\boldsymbol{\omega}) & =\sum_{\mathbf{n} \in \mathbb{Z}^{N}} d_{\mathbf{n}} \beta^{n}(\boldsymbol{\omega}-\mathbf{n}), \\
\forall \boldsymbol{\omega} & =\left(\omega_{1}, \ldots, \omega_{N}\right) \in \mathbb{R}^{N},
\end{aligned}
$$

where $d_{\mathbf{n}}$ are complex coefficients obtained by recursive digital filtering [31] of the samples $\hat{x}_{\mathbf{n}}$, independently for the real and imaginary part of the sequence $\hat{x}_{\mathbf{n}}$. There, $\hat{y}(\mathbf{n})=\hat{x}(\mathbf{n})$, and $\beta^{n}(\boldsymbol{\omega})$ is the $N$-dimensional B-spline of degree $n$ which is a separable function given by

$\beta^{n}(\boldsymbol{\omega})=\prod_{j=1}^{N} \beta^{n}\left(\omega_{j}\right)$,

where $\beta^{n}(\cdot)$ denotes the centered $\mathrm{B}$-spline of degree $n$ [29]. The separability makes possible that an operation on $N$-dimensional data is performed as a successive processing of one-dimensional (1D) data along each of the $N$ dimensions. In return, the data processing is simple and fast.

\subsubsection{Interpolation accuracy}

Let $f_{T}$ be the function that has been reconstructed from the samples of a function $f$, that is, $f_{T}(x)=\sum_{k \in \mathbb{Z}} c_{k} \varphi(x / T-k)$, where $T$ is the sampling step and where $c_{k}$ are coefficients computed from the samples $f_{k}$ using digital filtering [28]. We can define the error of approximation of $f$ by $f_{T}$ using the mean-square norm $\varepsilon^{2}(T)=\left\|f-f_{T}\right\|_{L_{2}}^{2}=$ $\int_{-\infty}^{\infty}\left(f(x)-f_{T}(x)\right)^{2} \mathrm{~d} x$. A prediction of this approximation error can be obtained using the formula $\eta^{2}(T)=\frac{1}{2 \pi} \int_{-\infty}^{\infty}|\hat{f}(\omega)|^{2} E(\omega T) \mathrm{d} \omega$ [32-34]. The interpolation error kernel $E$ depends on the basis function only. For bandlimited functions, we have that $\varepsilon$ is equal to $\eta$, and the prediction is exact in this case. 
The kernel $E$ must vanish at the origin to ensure that the approximation error vanishes for $T=0$. The vanishing rate is controlled by two properties of the basis function $\varphi$ : the approximation order $L$ and the approximation constant $C>0$. They determine $\eta$ as follows:

$\eta(T)=C T^{L}\left\|f^{(L)}\right\|_{L_{2}} \quad$ as $T \rightarrow 0$,

where $f^{(L)}$ is the $L$ th derivative of $f$. This means that the error predicted by $\eta$ decreases like $T^{L}$ as $T \rightarrow 0$. The higher is $L$, the faster is the decay of the approximation error for the same, sufficiently small $T$. Also, the smaller is $C$, the smaller is the approximation error. However, the decay of the approximation error is dominated by $T^{L}$ for $T \leqslant C$ which is very often the case. Therefore, $C$ is used for ranking basis functions of identical approximation order $L$. See [28] for a systematic overview of standard basis functions.

It turns out that the approximation order (and the support) of a B-spline of degree $n$ is equal to $L=n+1$ [28]. They have been shown to be maximally continuous basis functions, with the minimal support for a given order of approximation, and the maximal order of approximation for a given support [35].

\subsection{Multiresolution cost function}

We minimize the following cost function:

$$
\begin{aligned}
S(\mathbf{a})= & \frac{1}{2} \sum_{\mathbf{n} \in \mathbb{Z}_{*}^{2}} w(\mathbf{n})\left[\left(\frac{R_{\mathbf{a}}(\mathbf{n})}{\sigma_{\mathbf{a}}}-\frac{R(\mathbf{n})}{\sigma}\right)^{2}\right. \\
& \left.+\left(\frac{I_{\mathbf{a}}(\mathbf{n})}{\sigma_{\mathbf{a}}}-\frac{I(\mathbf{n})}{\sigma}\right)^{2}\right],
\end{aligned}
$$

where $\mathbb{Z}_{*}^{2}=\mathbb{Z}^{2} \backslash\{\mathbf{0}\}, R_{\mathrm{a}}$ and $I_{\mathrm{a}}$ are the real and imaginary part of the discrete version of (6), respectively, and where $R$ and $I$ are the real and imaginary part of the 2D DFT of the EM image, respectively.

EM images and projections have different ranges of intensities. To take this into account, we perform a normalization by their standard deviations, $\sigma$ and $\sigma_{\mathbf{a}}$, respectively, where $\sigma^{2}=$ $\sum_{\mathbf{n} \in \mathbb{Z}_{*}^{2}}\left(R^{2}(\mathbf{n})+I^{2}(\mathbf{n})\right), \quad$ and $\quad \sigma_{\mathbf{a}}^{2}=\sum_{\mathbf{n} \in \mathbb{Z}_{*}^{2}}\left(R_{\mathbf{a}}^{2}(\mathbf{n})+\right.$ $\left.I_{\mathbf{a}}^{2}(\mathbf{n})\right)^{*}$. We improve the robustness of the algorithm by weighting the cost function by a $2 \mathrm{D}$ Gaussian $w$. A coarse-to-fine processing can be performed by increasing the standard deviation of the Gaussian which increases the contribution of the higher frequencies to the cost function.

We adjust the standard deviations of the Gaussian functions, space-limiting and costweighting, such that a good registration accuracy can be achieved. In this paper, we have chosen these standard deviations as $\sigma_{g}=N / 2$, where the image size was $N \times N$ pixel and the volume size was $N \times N \times N$ voxel. The role of the costweighting function is to attenuate all frequencies, particularly the high ones; at the same time, we also discard the DC component at frequency $\boldsymbol{\omega}=$ 0. We can therefore say that the overall effect of our algorithm is a band-pass filtering. Note that the highest frequency is attenuated by approximately $40 \%$. The attenuation of the higher frequencies is beneficial because of a poor signalto-noise ratio (SNR) at these frequencies.

\subsection{Optimization}

\subsubsection{Levenberg-Marquardt optimizer}

We apply the LM algorithm to optimize the cost function. It achieves the best tradeoff between the robust but generally inefficient method of steepest descent and the efficient but non-robust Newton method [36,37]. This method uses the gradient $[\nabla S(\mathbf{a})]_{i}=\partial S / \partial a_{i}, i=1,2, \ldots, 5$ and the Hessian $\nabla^{2} S(\mathbf{a})$.

Let $H$ be a modified Hessian such that the diagonal elements of the Hessian are multiplied by a factor $(1+\lambda)$ while its off-diagonal elements are not changed

$[H(\mathbf{a})]_{i, j}=\left[\nabla^{2} S(\mathbf{a})\right]_{i, j}\left(1+\lambda \delta_{i, j}\right)$,

where Kronecker's delta is given by $\delta_{i, j}=1-$ $|\operatorname{sign}(i-j)|$ with $i, j \in\{1,2, \ldots, 5\}$. Then, the LM optimization algorithm can be described by

$\mathbf{a}^{(k+1)}=\mathbf{a}^{(k)}-\left(H\left(\mathbf{a}^{(k)}\right)\right)^{-1} \nabla S\left(\mathbf{a}^{(k)}\right)$.

Eq. (12) approximates the gradient algorithm for $\lambda \rightarrow+\infty$, albeit with vanishing steps. Similarly, it approximates the Newton algorithm for $\lambda \rightarrow 0$. The parameter $\lambda$ is adaptively tuned $[36,37]$ to provide a smooth transition from the 
steepest-descent algorithm (used in the beginning) to the Newton algorithm (used as approaching the solution).

\subsubsection{Gauss-Newton approximation of the Hessian}

The exact components of the true Hessian matrix depend on the first derivatives of $R_{\mathrm{a}}$ and $I_{\mathrm{a}}$ with respect to the parameters $\mathbf{a}$ as well as on their second derivatives. Since the influence of the second derivative terms can be destabilizing when the current guess is far from the solution, we use an approximation of the elements of the Hessian matrix that ignores them [37]. This approximation makes the optimization work better because it is necessarily positive-definite for a sufficiently large $\lambda$.

\subsubsection{Gradient}

The gradient of the cost function depends on the first derivatives of $R_{\mathrm{a}}$ and $I_{\mathrm{a}}$ with respect to the parameters a. To obtain these derivatives, we compute the gradient of the volume in the frequency domain

$\boldsymbol{\nabla} \hat{y}(\boldsymbol{\omega})=\sum_{\mathbf{n} \in \mathbb{Z}^{N}} d_{\mathbf{n}} \boldsymbol{\nabla} \beta^{n}(\boldsymbol{\omega}-\mathbf{n}), \quad \forall \boldsymbol{\omega} \in \mathbb{R}^{N}$.

For B-splines of degree $n \geqslant 2$, we have the guarantee that the first derivative $\mathrm{d} / \mathrm{d} x \beta^{n}(x)$ is continuous, which is not the case if $n=0$ (nearestneighbor interpolation) or $n=1$ (linear interpolation). We choose to model the data using cubic Bsplines $(n=3)$ since they provide a gradient of the cost function which is well-defined everywhere. At the same time, they offer a good tradeoff between computational cost and interpolation quality.

\section{Validation with ground-truth alignment}

In this section, we perform an objective evaluation of the accuracy of our algorithm using simulated EM images with a priori known ground-truth orientational and translational parameters. For this purpose, we have designed a measure of the assignment accuracy that we call a measure of misregistration and that we present in Section 4.1. As volumetric data, we use the models of two proteins from the PDB. To obtain the reference images, we simulate a set of cryo-EM images by projecting the 3D protein model with known orientational and translational parameters, by applying noise, and by simulating the effect of the CTF. The volumetric data and the procedure of synthesizing cryo-EM images are given in Section 4.2.

We performed four sets of experiments. The first set (Section 4.3) tests the robustness of the algorithm with respect to the initial values for the parameters a. We compare the robustness of the frequency-based algorithm to that of the spacebased method [24,25] on the same EM data set. The second set (Section 4.4) was designed to investigate which quantized-parameter method to apply for the initial assignment. It also tests the "mixed" strategy based on several different initializations. The third set (Section 4.5) tests the sensitivity of our algorithm when initialized by MPM to the library quantization step. The fourth set (Section 4.6) tests the influence of the quality of the 3D reference model on the performance of the algorithm. We have therefore low-pass filtered the model used for the robustness experiment and repeated it for the same initial conditions.

\subsection{Measure of misregistration}

We transform the 3D index of each voxel of a virtual volume, given in homogenous coordinates $\mathbf{m}=\left(m_{1}, m_{2}, m_{3}, 1\right)$, by using two transformations: the ground-truth transformation $B$ and the estimated transformation $B_{\mathrm{a}}^{\alpha}$. The transformation matrices $B$ and $B_{\mathbf{a}}^{\alpha}$ are of size $4 \times 4$ (homogenous coordinates) and comprise rotation, translation, and scale parameters. The transformation $B_{\mathrm{a}}^{\alpha}$ takes into account the symmetries of the particular protein. They involve a rotation by an angle $\alpha$ around the symmetry axis before applying the transformation parameters a estimated by imageto-volume registration. In the case of a protein with a threefold rotational symmetry, $\alpha$ takes the following values: $60^{\circ}, 120^{\circ}$, and $240^{\circ}$. We compute the average of the norm of the difference between the two transformed coordinates over all $\mathbf{m}$ for each $\alpha$. We define the misregistration as the 
minimum averaged norm, that is,

$\varpi=\min _{\alpha} \frac{1}{\operatorname{card}(f)} \sum_{\mathbf{m} \in f}\left\|\left(B-B_{\mathbf{a}}^{\alpha}\right) \mathbf{m}\right\|$.

A smaller $\varpi$ indicates a more-accurate angular and translational assignment. The perfect assignment should result in $\varpi=0$. Note that this measure is expressed in voxel units but takes into account both angular and translational assignment accuracy.

\subsection{Data}

\subsubsection{Volumes}

The Bacteriorhodopsin (PDB accession code: 1BRD, Fig. 5(a)), found in Halobacterium halobium, has been solved using high-resolution cryoEM [38]. The Adenovirus Type 5 Hexon (PDB accession code: 1RUX, Fig. 5(b)), found in humans, has been solved by X-ray crystallography [39]. Each protein has a threefold rotational symmetry. The two volumes were of size $64 \times$ $64 \times 64$ voxel and their sampling rate was $3.27 \AA$.

\subsubsection{Synthetic EM images}

1000 projections of the reference model were computed with random orientation determined by a set of uniformly distributed angles $\varphi \in\left[0^{\circ}, 120^{\circ}\right]$ and $\theta \in\left[0^{\circ}, 175^{\circ}\right]$. The three remaining parameters
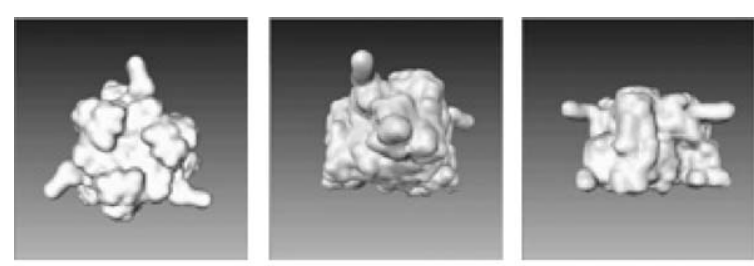

(a)
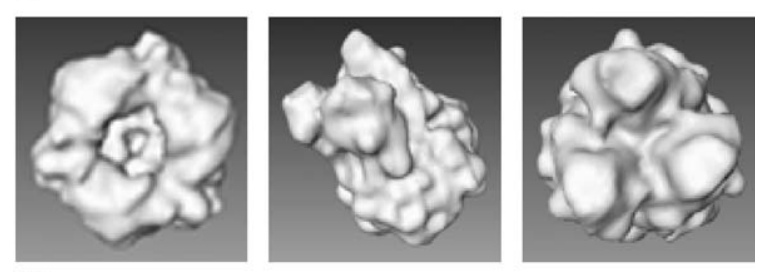

(b)

Fig. 5. Three views of an isosurface of (a) the Bacteriorhodopsin and (b) the Hexon from the PDB. $\psi, \Delta x$, and $\Delta y$ were equal to zero for each projection.

Noise and the CTF were simulated using a model proposed by Velázquez-Muriel et al. [40]. The noise before the CTF $\left(n_{\mathrm{b}}\right)$ was modeled by a Gaussian white noise with the standard deviation $\sigma_{\mathrm{b}}=0.54$. The noise after the $\operatorname{CTF}\left(n_{\mathrm{a}}\right)$ was modeled by a low-pass filtered Gaussian noise with the standard deviation $\sigma_{\mathrm{a}}=2.20$ generated at the input $n_{\mathrm{a}}^{\prime}$. The standard deviations were adjusted so that the ratio between the power of the signal and the power of the total noise was approximately equal to $\frac{1}{3}$. The filter and the CTF have been estimated simultaneously [40] from an electron micrograph for the following parameters of the electron microscope: accelerating $\quad$ voltage $=200 \mathrm{kV}, \quad$ defocus $=-27700 \AA$, spherical aberration $=2 \mathrm{~mm}$, convergence cone $=$ $0.21 \mathrm{mrad}$. The CTF, the background spectrum filter, and the synthesized EM images were of size $64 \times 64$ pixel. The synthesizing procedure is schematically shown in Fig. 6.

\subsection{Robustness}

We performed four experiments using the Bacteriorhodopsin data. In the first experiment, we initialized the parameters by their ground-truth values. In the second experiment, we initialized the angles by their ground-truth values modified by $\pm 5^{\circ}$ with probability 0.5 . The initial values for the translation parameters were equal to their groundtruth values. In the third and fourth experiments, the angular perturbation was $\pm 10^{\circ}$ and $\pm 15^{\circ}$, respectively.

The mean values and the standard deviations of the initial misregistration and of the misregistration after having performed the spaceand frequency-based registrations are shown in Table 1. Starting from the ground-truth assignment, the two algorithms performed in a similar way (with a confidence of $95 \%$ ). Both algorithms converged to an assignment that was not the ground-truth one. This can be explained by the presence of noise and of the CTF, which makes a synthetic EM image differ from the corresponding theoretical projection. In all three cases of angular perturbation, both algorithms were successful in 


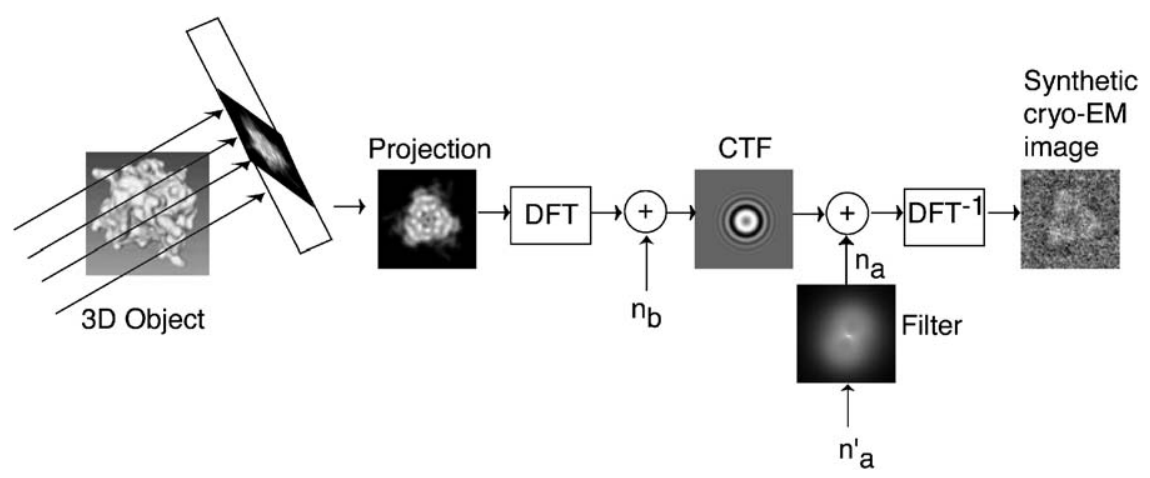

Fig. 6. Procedure for synthesizing EM images.

Table 1

Robustness of the space- and frequency-based algorithm

\begin{tabular}{|c|c|c|c|c|c|c|}
\hline & \multicolumn{6}{|c|}{ Misregistration [voxel] } \\
\hline & \multicolumn{2}{|l|}{ Initial } & \multicolumn{2}{|c|}{ Space-based } & \multicolumn{2}{|c|}{ Frequency-based } \\
\hline & Mean & $\begin{array}{l}\text { Standard } \\
\text { deviation }\end{array}$ & Mean & $\begin{array}{l}\text { Standard } \\
\text { deviation }\end{array}$ & Mean & $\begin{array}{l}\text { Standard } \\
\text { deviation }\end{array}$ \\
\hline Ground truth & 0 & 0 & 1.25 & 0.58 & 1.30 & 0.77 \\
\hline Ground truth $\pm 5^{\circ}$ & 3.55 & 0.89 & 1.89 & 0.77 & 1.61 & 0.85 \\
\hline Ground truth $\pm 10^{\circ}$ & 7.10 & 1.76 & 4.30 & 2.22 & 1.83 & 1.31 \\
\hline Ground truth $\pm 15^{\circ}$ & 10.55 & 2.61 & 7.86 & 3.93 & 2.53 & 2.91 \\
\hline
\end{tabular}

reducing the initial misregistration. For the angular perturbation of $\pm 5^{\circ}$, the frequency-based method was slightly more accurate (with a confidence of $99.9 \%$ ) than the space-based method. In the two remaining cases, $\pm 10^{\circ}$ and $\pm 15^{\circ}$, the frequency-based method was much more accurate (with a confidence of $99.9 \%$ ) than the space-based method.

The synthesized images were very similar to the data commonly encountered in 3D cryo-EM. Since they were nearly space-limited, their accurate interpolation in the Fourier domain could be achieved using sinc-like basis functions. Our algorithms are based on cubic-spline interpolation. The cardinal cubic spline is quite similar to the sinc function [29]. We observe that the frequency-based registration outperforms the space-based method. We explain this result by the fact that the spacelimitedness assumption is better satisfied by the data at hand than the band-limitedness assumption implicit with real-space data interpolation.

\subsection{Refinement of a previous assignment}

Here, we show the performance of the frequency-based method when initialized by MPM, PM, or RA assignments. We also show their joint result based on the minimum final value of the dissimilarity measure among the three methods.

MPM and PM methods used a library of 1704 images uniformly distributed in the $3 \mathrm{D}$ space with an angular step of $5^{\circ}$. The RT was also sampled every $5^{\circ}$. The results of this experiment for the Bacteriorhodopsin and the Hexon are shown in Tables 2 and 3, respectively. From these tables, we observe that our algorithm was successful in reducing (with $99.9 \%$ confidence) the initial misregistration coming from the MPM and PM 
Table 2

Accuracy of the frequency-based algorithm for three initial assignments in the Bacteriorhodopsin case, as well as their joint result

\begin{tabular}{lccccc}
\hline $\begin{array}{l}\text { Initial } \\
\text { guess } \mathbf{a}_{0}\end{array}$ & \multicolumn{2}{l}{$\begin{array}{l}\text { Initial misregistration } \\
\text { [voxel] }\end{array}$} & & \multicolumn{2}{l}{$\begin{array}{l}\text { Final misregistration } \\
\text { [voxel] }\end{array}$} \\
\cline { 2 - 3 } \cline { 5 - 6 } & Mean & $\begin{array}{r}\text { Standard } \\
\text { deviation }\end{array}$ & & Mean & $\begin{array}{r}\text { Standard } \\
\text { deviation }\end{array}$ \\
\hline MPM & 3.18 & 1.85 & & 1.77 & 1.11 \\
PM & 2.99 & 3.68 & & 1.70 & 1.54 \\
RA & 16.55 & 18.80 & & 15.83 & 19.56 \\
MPM- & N/A & N/A & & 1.70 & 1.06 \\
PM-RA & & & & \\
\hline
\end{tabular}

Table 3

Accuracy of the frequency-based algorithm for three initial assignments in the Hexon case, as well as their joint result

\begin{tabular}{lccccc}
\hline $\begin{array}{l}\text { Initial } \\
\text { guess } \mathbf{a}_{0}\end{array}$ & \multicolumn{2}{l}{$\begin{array}{l}\text { Initial misregistration } \\
\text { [voxel] }\end{array}$} & & \multicolumn{2}{l}{$\begin{array}{l}\text { Final misregistration } \\
\text { [voxel] }\end{array}$} \\
\cline { 2 - 3 } \cline { 5 - 6 } & Mean & $\begin{array}{l}\text { Standard } \\
\text { deviation }\end{array}$ & & Mean & $\begin{array}{r}\text { Standard } \\
\text { deviation }\end{array}$ \\
\hline MPM & 4.50 & 6.86 & & 3.38 & 7.64 \\
PM & 9.13 & 14.05 & & 8.91 & 15.62 \\
RA & 35.00 & 11.59 & & 33.70 & 14.90 \\
MPM- & N/A & N/A & 1.97 & 3.57 \\
PM-RA & & & & \\
\hline
\end{tabular}

assignments in the Bacteriorhodopsin case and from the MPM assignment in the Hexon case. We also see that it fails to register data that are initially too much misregistered as in case of the RA assignment for both proteins and of the PM assignment for the Hexon.

We notice that the strategy that involves all three methods improves the accuracy of our algorithm for both proteins. For these data sets, it achieves registration with a mean accuracy that is better than 2 voxel (i.e., $6.54 \AA$ ). In these examples, it has rarely selected the starting points coming from RA, which shows an improved robustness. By help of the next two figures, we investigate the quality of this strategy using the Hexon data. In Fig. 7, we show the goodness of the cost function as a selection criterion by

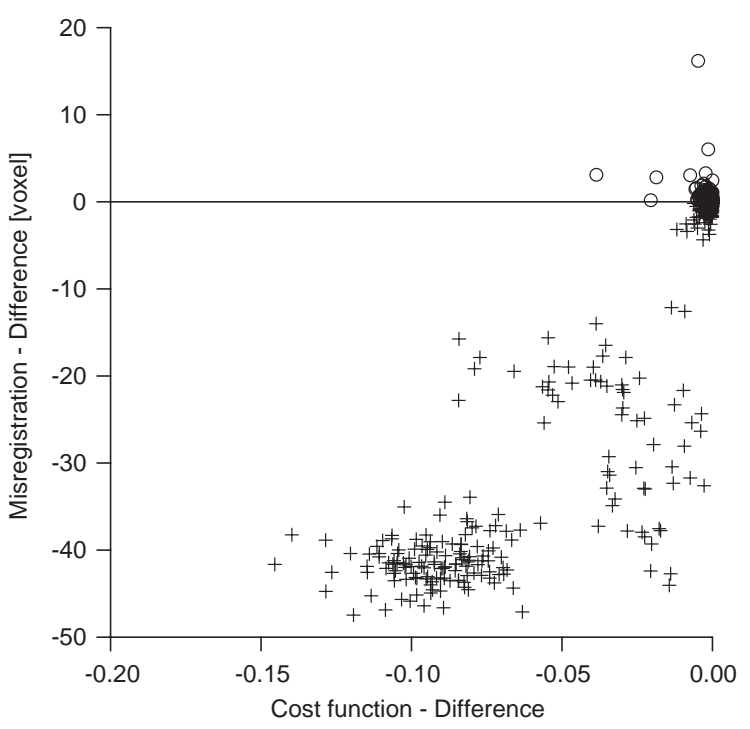

Fig. 7. A joint result of MPM, PM, and RA assignments of the frequency-based algorithm in the Hexon case. Difference between the two lowest-cost functions vs. difference between the corresponding misregistrations.

plotting the difference between the two lowestcost functions vs. the difference between the corresponding objective misregistrations. In the cases where both differences are negative, the strategy was right. We see that, because of the noise, it failed in a small region where the difference between the cost functions was close to 0 . In most cases, the difference between the corresponding misregistrations was small which means that the assignment error was small too. Fig. 8 shows the initial and final joint misregistrations. We observe that, using this strategy, our algorithm reduces any joint misregistration, as long as it is lower than about 25 voxel.

As far as speed is concerned, for the image size $64 \times 64$ pixel and for the volume size $64 \times 64 \times 64$ voxel, our registration takes about $12 \mathrm{~s}$ on a Power Mac G4, 733 MHz.

\subsection{Robustness to the angular step of the library}

We explored the effect of a coarser library of projections, used by MPM, on the performance of our frequency-based algorithm. We created a library of 150 projections of the Bacteriorhodopsin 


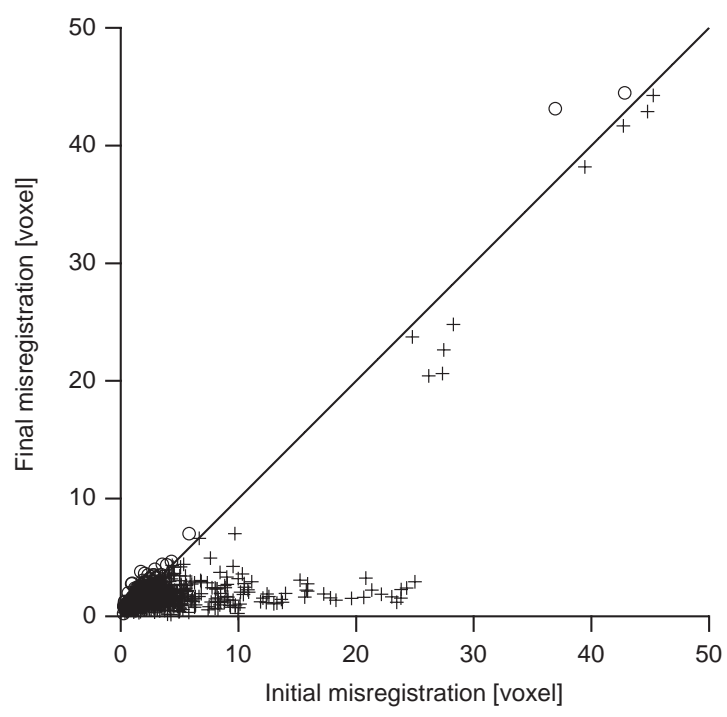

Fig. 8. A joint result of MPM, PM, and RA assignments of the frequency-based algorithm in the Hexon case. Final vs. initial misregistration corresponding to the lowest-cost function.

Table 4

Effect of a $5^{\circ}$ library and a $10^{\circ}$ library of the volume projections, used for the initial MPM, on the performance of the frequency-based algorithm

\begin{tabular}{llllll}
\hline Bacteriorhodopsin & $\begin{array}{l}5^{\circ} \text { library } \\
\text { Misregistration } \\
\text { [voxel] }\end{array}$ & & \multicolumn{2}{l}{$\begin{array}{l}10^{\circ} \text { library } \\
\text { Misregistration } \\
\text { [voxel] }\end{array}$} \\
\cline { 2 - 3 } \cline { 5 - 6 } & Mean & $\begin{array}{l}\text { Standard } \\
\text { deviation }\end{array}$ & & Mean & $\begin{array}{l}\text { Standard } \\
\text { deviation }\end{array}$ \\
\hline Initial & 3.18 & 1.85 & & 3.85 & 3.68 \\
Final & 1.77 & 1.11 & & 1.81 & 1.44 \\
\hline
\end{tabular}

with an angular step of $10^{\circ}$. Then, we performed the assignment using MPM. We run our algorithm with the volume and the images initially misregistered by $3.85 \pm 3.68$ voxel. The registration was achieved with an accuracy of $1.81 \pm 1.44$ voxel.

We notice that the initial misregistration was significantly higher (with $99.9 \%$ confidence) than the misregistration used to initialize the registration based on the $5^{\circ}$ library (Table 4 ). In both cases, we significantly reduced (with $99.9 \%$ confidence) the initial misregistration. However, the two assignments are not significantly different (with $95 \%$ confidence) (Table 4 ), which means that we can initialize our algorithm with the assignment based on a coarser library of projections without any significant loss in accuracy.

\subsection{Quality of the reference}

We have low-pass filtered the 3D Bacteriorhodopsin model described in Section 4.2 to $2.4 \mathrm{~nm}$ and repeated the robustness experiment (Section 4.3). We compare in Table 5 the results of the experiment with the low- and the high-resolution reference volumes for the same initial conditions. When the initial assignment is different from the ground truth, the algorithm reduces the initial misregistration in the case of the low- and of the high-resolution volume. For bigger initial misalignments (ground truth $\pm 10^{\circ}$, ground truth $\pm 15^{\circ}$ ), we can note a smaller standard deviation of the measure of misregistration in the case of the low-resolution volume than in the case of the highresolution one. This can be explained by a better convergence toward a global minimum of the cost function in the case of a low-resolution volume.

\section{Validation without ground-truth alignment}

Given a set of experimental images of a GroEL chaperonin from Escherichia coli and its lowresolution 3D model, we show here the performance of our algorithm in refining this model. In this case, the ground-truth orientation and translation of the particle images are unknown and have to be estimated. This means that we cannot perform an objective evaluation of the accuracy of the assignment anymore. Therefore, we have chosen to evaluate the performance of our algorithm by measuring the consistency of volume reconstructions in terms of the Fourier-shell correlation (FSC).

We had at our disposal a set of experimental GroEL images acquired by De Carlo et al. [41] and a first estimation of their orientational and translational parameters. This first assignment has been obtained after having performed one iteration of PM for refinement of a starting model (the zero-iteration model), as explained in Ref. [41]. Using these data, we reconstructed a 3D 
Table 5

Robustness of the frequency-based algorithm for two reference 3D models of different resolutions

\begin{tabular}{|c|c|c|c|c|c|c|}
\hline & \multicolumn{6}{|c|}{ Misregistration [voxel] } \\
\hline & \multicolumn{2}{|l|}{ Initial } & \multicolumn{2}{|c|}{ Low resolution } & \multicolumn{2}{|c|}{ High resolution } \\
\hline & Mean & $\begin{array}{l}\text { Standard } \\
\text { deviation }\end{array}$ & Mean & $\begin{array}{l}\text { Standard } \\
\text { deviation }\end{array}$ & Mean & $\begin{array}{l}\text { Standard } \\
\text { deviation }\end{array}$ \\
\hline Ground truth & 0 & 0 & 1.98 & 1.13 & 1.30 & 0.77 \\
\hline Ground truth $\pm 5^{\circ}$ & 3.55 & 0.89 & 2.26 & 1.05 & 1.61 & 0.85 \\
\hline Ground truth $\pm 10^{\circ}$ & 7.10 & 1.76 & 2.40 & 1.14 & 1.83 & 1.31 \\
\hline Ground truth $\pm 15^{\circ}$ & 10.55 & 2.61 & 2.70 & 1.87 & 2.53 & 2.91 \\
\hline
\end{tabular}

GroEL model that had a very low resolution. We refer to this volume as the first-iteration model.

We performed one iteration of the refinement of the first-iteration model using MPM. We refer to the subsequent reconstructed volume as the second-iteration model. In the next iteration, we applied our spline frequency-based algorithm to the refinement of the second-iteration model. To provide our algorithm with an initial angular and translational assignment, we performed one iteration of MPM that was also using the seconditeration model as the reference.

Using the assignment by our algorithm, we reconstructed two volumes from two subsets of the GroEL images, and computed the corresponding FSC curve. The 0.5 -FSC for the two volumes was $12.5 \AA$ (Fig. 9). We reconstructed two more volumes corresponding to the initial angular and translational assignment used by our algorithm, and computed the corresponding FSC curve. The 0.5 -FSC for these two volumes was $22.2 \AA$ (Fig. 9). We also had at our disposal the angular and translational assignment for the images after having performed four iterations of PM for refinement of the zero-iteration model, as explained in Ref. [41]. We therefore reconstructed two volumes corresponding to this assignment, and computed the FSC curve. Their 0.5-FSC was $29.0 \AA$ A(Fig. 9). All six volume reconstructions were accomplished using the algebraic reconstruction technique (ART) with smooth spherically symmetric volume elements [42]. From Fig. 9, we conclude that our spline frequency-based algorithm improves the consistency of $3 \mathrm{D}$ reconstructions. It

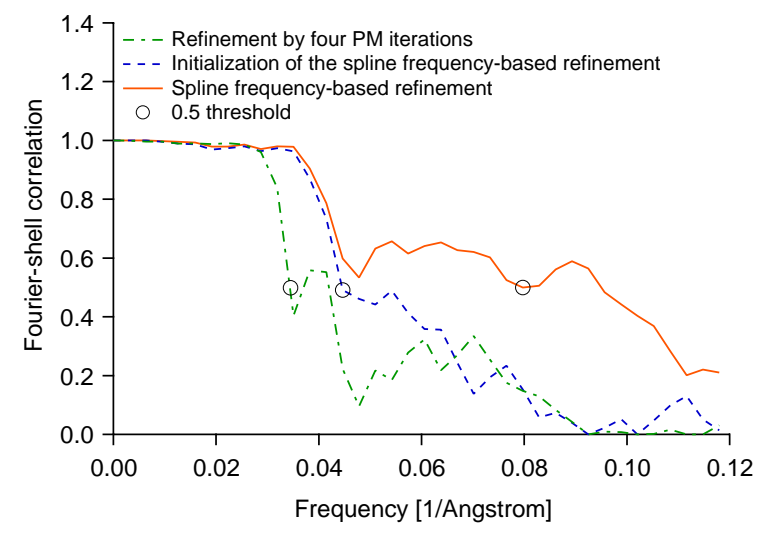

Fig. 9. Fourier-shell correlation and 0.5 threshold for the following GroEL volume reconstructions: (1) corresponding to the assignment by the spline frequency-based algorithm for refinement of the second-iteration model, (2) corresponding to the initialization of the spline frequency-based algorithm by MPM that was using the same 3D reference, that is, the seconditeration model, and (3) corresponding to the assignment after four iterations of PM for refinement of the zero-iteration model.

outperforms PM-based refinement procedure in terms of both consistency and speed; it leads to more consistent $3 \mathrm{D}$ reconstructions in a smaller number of iterations (Fig. 10).

\section{Conclusion}

We have developed a frequency-based algorithm for computing the parameters of orientation and translation of cryo-EM single-particle images with respect to a $3 \mathrm{D}$ model of the particle. Our 


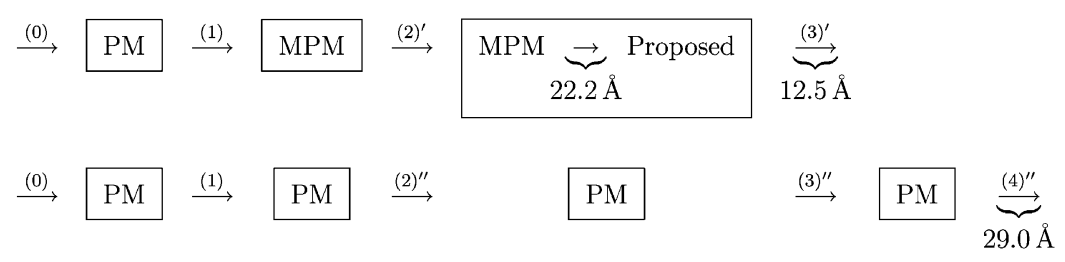

Fig. 10. Experiments performed, with the reconstruction index in parentheses, and with the corresponding FSC resolution. For the proposed method, the effective model is that which results from reconstruction (2)', and we use the assignments resulting from MPM only as initial conditions. (The reconstruction at $22.2 \AA$ was performed solely for monitoring purposes.) We reach a better resolution in fewer reconstructions than with the PM method.

algorithm assigns a set of continuous orientational and translational parameters to the images. Therefore, its application is the refinement of the assignment obtained by the algorithms that result in a set of quantized orientational and translational parameters. Our algorithm uses a gradientbased iterative minimization of a least-squares measure of dissimilarity between an image and a projection of the volume in the frequency domain. It is based on the CST for computing FT of the projections. To interpolate the data accurately and to compute the gradient of the dissimilarity measure exactly, we use a continuous data model based on cubic B-splines.

We validated the algorithm in a fully controlled simulation environment where the ground-truth solution was known a priori. We synthesized a set of images with known orientations and translations using a 3D model of a protein from the PDB. We designed a measure of the assignment quality that is expressed in voxel units but takes into account both angular and translational assignment accuracy.

We compared the robustness of this algorithm to that of an algorithm that performs the registration in the space domain. We observed that the frequency-based registration was more robust than the space-based method, which we explain by the space-limitedness assumption that is better satisfied by the EM data than the band-limitedness assumption implicit with real-space data interpolation.

Then, we showed the performance of the frequency-based method when initialized by applying one of three standard quantized-parameter methods. We also showed their joint result obtained by employing a strategy that selects for each image the method that gives the minimum final value of the dissimilarity measure. This strategy improves the robustness of our algorithm; for the data sets used here, it achieves assignment with a mean error that is smaller than 2 voxel (i.e., $6.54 \AA$ ).

Our experiments point out that we could initialize our algorithm with an assignment based on a $10^{\circ}$ library of projections without any significant loss in accuracy with respect to an assignment based on a $5^{\circ}$ library. This good robustness suggests a strategy where a coarse initialization is performed using any of a series of quantized-parameter algorithms, followed by our continuous-parameter approach.

We also tested the influence of the quality of the $3 \mathrm{D}$ reference model to the performance of the algorithm. We have low-pass filtered the model used for the robustness experiment and repeated the robustness experiment with the lower-quality model. When the initial assignment is different from the ground truth, the algorithm reduces the initial misregistration with the low-resolution volume like with the high-resolution reference. We noticed once more that robustness to local minima of the cost function was better in the case of a low-resolution reference than in the case of a high-resolution one.

Finally, we presented the performance of our frequency-based algorithm in refining a 3D model of a GroEL chaperonin using real EM data where there was no ground-truth solution. We initialized it by MPM. We observed that our algorithm improved the consistency of the volumes from a previous iteration and outperformed PM-based 
assignment in terms of consistency and speed. In particular, we observed on this data that our proposed approach was able to reach, in three iterations, a better 0.5-FSC accuracy $(12.5 \AA)$ than that achieved by four iterations of PM $(29.0 \AA)$.

Our frequency-based method is available in the Xmipp package [43].

\section{References}

[1] J. Frank, Three-Dimensional Electron Microscopy of Macromolecular Assemblies, Academic Press, New York, 1996.

[2] J. Frank, Annu. Rev. Biophys. Biomol. Struct. 31 (2002) 303.

[3] W. Kühlbrandt, K.A. Williams, Curr. Opin. Chem. Biol. 3 (1999) 537.

[4] V.M. Unger, Curr. Opin. Struct. Biol. 11 (2001) 548.

[5] S. Basu, Y. Bresler, IEEE Trans. Image Process. 9 (2000) 1107.

[6] S. Basu, Y. Bresler, IEEE Trans. Image Process. 9 (2000) 1094.

[7] J. Dengler, Ultramicroscopy 30 (1989) 337.

[8] M.S. Gelfand, A.B. Goncharov, Transl. Math. Monographs 81 (1990) 97.

[9] A.B. Goncharov, Transl. Math. Monographs 81 (1990) 67.

[10] N. Grigorieff, J. Mol. Biol. 277 (1998) 1033.

[11] G. Harauz, F.P. Ottensmeyer, Ultramicroscopy 12 (1984) 309.

[12] P.D. Lauren, N. Nandhakumar, IEEE Trans. Pattern Anal. Mach. Intell. 19 (1997) 417.

[13] P.A. Penczek, R.A. Grassucci, J. Frank, Ultramicroscopy 53 (1994) 251.

[14] P.A. Penczek, J. Zhu, J. Frank, Ultramicroscopy 63 (1996) 205.

[15] M. Radermacher, Ultramicroscopy 53 (1994) 121.

[16] D.B. Salzman, Comput. Vision Graphics Image Process. $50(1990) 129$.

[17] C.O.S. Sorzano, S. Jonić, C. El-Bez, J.M. Carazo, S. De Carlo, P. Thévenaz, M. Unser, J. Struct. Biol. 146 (2004) 381.

[18] M. van Heel, B. Gowen, R. Matadeen, E.V. Orlova, R. Finn, T. Pape, D. Cohen, H. Stark, R. Schmidt, M. Schatz, A. Patwardhan, Quart. Rev. Biophys. 33 (2000) 307.

[19] A.C. Kak, M. Slaney, Principles of Computerized Tomographic Imaging, IEEE Press, New York, 1988.

[20] F. Natterer, The Mathematics of Computerized Tomography, Wiley and B G Teubner, Stuttgart, 1986.
[21] F. Natterer, F. Wübbeling, Mathematical Methods in Image Reconstruction, Philadelphia, PA, Society for Industrial and Applied Mathematics, 2001.

[22] S.W. Provencher, R.H. Vogel, Ultramicroscopy 25 (1988) 209.

[23] R.H. Vogel, S.W. Provencher, Ultramicroscopy 25 (1988) 223.

[24] S. Jonić, P. Thévenaz, M. Unser, Multiresolution splinebased $3 \mathrm{D} / 2 \mathrm{D}$ registration of $\mathrm{CT}$ volume and $\mathrm{C}$-arm images for computer-assisted surgery, in: M. Sonka, K.M. Hanson (Eds.), Proceedings of SPIE, Medical Imaging 2001: Image Processing, vol. 4322, 2001, pp. 1101-1109.

[25] S. Jonić, P. Thévenaz, M. Unser, Multiresolution-based registration of a volume to a set of its projections, in: $\mathrm{M}$. Sonka, J.M. Fitzpatrick (Eds.), Proceedings of SPIE, Medical Imaging 2003: Image Processing, vol. 5032, 2003, pp. 1049-1052.

[26] A. Aldroubi, M. Unser, M. Eden, Signal Process. 28 (1992) 127.

[27] I.J. Schoenberg, Quart. Appl. Math. 4 (1946) 45-112.

[28] P. Thévenaz, T. Blu, M. Unser, IEEE Trans. Med. Imaging 19 (2000) 739.

[29] M. Unser, IEEE Signal Process. Mag. 16 (1999) 22.

[30] H.M. Berman, J. Westbrook, Z. Feng, G. Gilliland, T.N. Bhat, H. Weissig, I.N. Shindyalov, P.E. Bourne, Nucleic Acids Res. 28 (2000) 235.

[31] M. Unser, A. Aldroubi, M. Eden, IEEE Trans. Signal Process. 41 (1993) 834.

[32] T. Blu, M. Unser, Appl. Comput. Harmonic Anal. 6 (2) (1999) 219.

[33] T. Blu, M. Unser, IEEE Trans. Signal Process. 47 (10) (1999) 2783.

[34] T. Blu, M. Unser, IEEE Trans. Signal Process. 47 (10) (1999) 2796.

[35] T. Blu, P. Thévenaz, M. Unser, IEEE Trans. Image Process. 10 (2001) 1069.

[36] C.T. Kelley, Iterative Methods for Optimization, Society for Industrial and Applied Mathematics, Philadelphia, PA, 1999.

[37] W. Press, B. Flannery, S. Teukolsky, W. Vetterling, Numerical Recipes, The Art of Scientific Computing, Cambridge University Press, Cambridge, 1988.

[38] R. Henderson, J.M. Baldwin, T.A. Ceska, F. Zemlin, E. Beckmann, K.H. Downing, J. Mol. Biol. 213 (1990) 899.

[39] J.J. Rux, R.M. Burnett, Mol. Therapy 1 (2000) 18.

[40] J.A. Velázquez-Muriel, C.O.S. Sorzano, J.J. Fernández, J.M. Carazo, Ultramicroscopy 96 (2003) 17.

[41] S. De Carlo, C. El-Bez, C. Alvarez-Rúa, J. Borge, J. Dubochet, J. Struct. Biol. 138 (2002) 216.

[42] R. Marabini, G.T. Herman, J.M. Carazo, Ultramicroscopy 72 (1998) 53.

[43] R. Marabini, I.M. Masegosa, S.M.M.C. SanMartín, J.J. Fernández, L.G. de la Fraga, C. Vaquerizo, J.M. Carazo, J. Struct. Biol. 116 (1996) 237. 